\title{
Computation of Electromagnetic Fields in Assemblages of Biological Cells Using a Modified Finite-Difference Time-Domain Scheme
}

\author{
Chan H. See, Raed A. Abd-Alhameed, and Peter S. Excell, Senior Member, IEEE
}

\begin{abstract}
When modeling objects that are small compared with the wavelength, e.g., biological cells at radio frequencies, the standard finite-difference time-domain (FDTD) method requires extremely small time-step sizes, which may lead to excessive computation times. The problem can be overcome by implementing a quasi-static approximate version of FDTD based on transferring the working frequency to a higher frequency and scaling back to the frequency of interest after the field has been computed. An approach to modeling and analysis of biological cells, incorporating a generic lumped-element membrane model, is presented here. Since the external medium of the biological cell is lossy material, a modified Berenger absorbing boundary condition is used to truncate the computation grid. Linear assemblages of cells are investigated and then Floquet periodic boundary conditions are imposed to imitate the effect of periodic replication of the assemblages. Thus, the analysis of a large structure of cells is made more computationally efficient than the modeling of the entire structure. The total fields of the simulated structures are shown to give reasonable and stable results at 900,1800 , and $2450 \mathrm{MHz}$. This method will facilitate deeper investigation of the phenomena in the interaction between electromagnetic fields and biological systems.
\end{abstract}

Index Terms-Finite difference time domain (FDTD), Floquet periodic boundary conditions, quasi-static method.

\section{INTRODUCTION}

$\mathbf{R}$ ESEARCH INTO possible mechanisms of interaction of electromagnetic (EM) fields with biological tissues and cells in culture has motivated a growing need for accurate models describing the EM behavior of cells exposed to these fields. Therefore, several numerical models have been created in order to study the interaction between EM fields and biological entities, at the tissue, cell, and ionic levels. In this area, the most frequently used technique for computing the EM field is the finite-difference time-domain (FDTD) method [1], [2] due to its independence from the material parameters.

The original FDTD method requires extremely small time-step sizes when modeling electrically small regions (much smaller than a wavelength): this is especially the case when modeling biological cells since they have maximum dimensions of a few tens of micrometers. Thus, it can become impractical due to the unaffordable computation times required. This problem can be solved by implementing a quasi-static approximate version of FDTD. This approach is based on transferring

Manuscript received August 4, 2006; revised March 24, 2007.

The authors are with the Mobile and Satellite Communication Research Centre, University of Bradford, Bradford BD7 1DP, U.K. (e-mail: c.h.see1@brad.ac.uk; r.a.a.abd@brad.ac.uk; p.s.excell@brad.ac.uk).

Digital Object Identifier 10.1109/TMTT.2007.904064 the working frequency to a higher frequency to reduce the number of time steps required. The generated internal field at the higher frequency can then be scaled back to the frequency of interest [3]-[6].

Cells are surrounded by thin membranes, typically a few nanometers thick [7]. They are the major barrier in the cell, separating the inside of the cell from the exterior medium. It is this structure that allows cells to selectively interact with their environment. Therefore, the cell membrane has been identified as the primary target for the study of possible actions of EM fields on biological structures. Since the thickness of the membrane is approximately 1000 times smaller than the width of a typical biological cell, if the standard FDTD procedure were to be blindly applied to model detail in the membrane within a complete cell model, this would cause some millions of iterations to be required to complete one cycle of simulation. This again would cause excessive computation time. To overcome this drawback in standard FDTD, the lumped-element FDTD method [8]-[11] was implemented to model the behavior of the membrane. A generic long-established model (the Hodgkin-Huxley (HH) model [12]-[16]) was used to demonstrate the functionality of this method: it is known that the $\mathrm{HH}$ model is imperfect for microwave frequencies, but it is reasonable to use it as a working hypothesis (as have others [4]) to develop modeling techniques while operational versions of improved models are still in development.

For the sake of simplicity, the analyzed structure has been represented with spherical or cubical cells, and Floquet periodic boundary conditions [17]-[20] have been applied to the border of the analyzed structure in order to mimic the presence of the surrounding cells. Although cellular tissues are not perfectly periodic and living cells are not precisely spheres or cubes, these approximations allow a reasonable approach to the modeling of biological tissue using only a small part of the structure, while alleviating the problem of the huge requirement of computer resources for the simulation of a complete body of tissue. Since the external medium of the biological tissue is lossy fluid, the modified Berenger perfectly matched layer (PML) absorbing boundary condition [21]-[24] is used to truncate the computation grid, in order to reduce the reflections on the interface layers: this is more accurate than the Mur boundary condition [25], [26] used in other recent work [4].

A further difficulty is the limited extent of studies on the dielectric properties of cell tissues [27]; thus, the complex permittivity of each cell tissue is not clearly established for radio frequencies. However, in this study, an analytical method for estimating the electrical properties of cell tissues in the RF band 
[28] will be adopted throughout the analysis. Earlier work only considered two media (water and membrane) [4], but the procedure adopted here enables the tissue model to consist of three media (lossy medium, membrane, and cytoplasm). In addition, a mass of connected biological tissue is simulated by creating an equivalent stack of compacted cells (both spherical, with interstices, and fully compacted cubical). The total electric fields along the central axes of rows of these spherical and cubical cellular structures will be investigated.

\section{SUMMARY OF MethoD}

\section{A. Quasi-Static FDTD Scheme}

The interaction between animals and humans exposed to extremely low-frequency electric fields was investigated by Kaune and Gillis [29] and Guy et al. [30] in 1981 and 1982, respectively. Their research results furnish valuable analytical and experimental verification of the concept of quasi-static coupling at power-line frequencies. Later authors [5], [6], [31] implemented the same principles using FDTD to study the numerical dosimetry of anatomically based models. Recently, the same idea was further extended to modeling the interaction between EM fields and biological tissue at mobile communication frequencies, i.e., GSM900 and GSM1800 [4].

In order to implement the quasi-static approximation to analyze scattering problems, the maximum dimension of the structure under investigation must be less than approximately onetenth of the wavelength in the surrounding medium [29], [30]. According to the scaling relationship between the fields at frequencies $f$ and $f^{\prime}$ that was derived in [5] and [30], a higher working frequency $\left(f^{\prime}\right)$ that still falls within the quasi-static regime can be chosen to excite the model to reduce the computation time.

\section{B. Modified Berenger PML}

The PML, introduced by Berenger [21] in 1994, allowed boundary reflections below $-80 \mathrm{~dB}$ to be realized. The PML is based on surrounding the FDTD problem space with a highly lossy and matched nonphysical absorber. It has been found to be the most accurate technique of the absorbing boundary conditions available and has become standard in most current FDTD implementations [32]. For the case of PML layers with conductivities increasing geometrically, the geometric grading factor $(g)$ can be modified in order to reduce the reflection on the interface layer when the problem space is entirely within a lossy-medium environment. An empirical expression from which $g$ can be found, and which has been found to give good results [23], [24] is

$$
\sigma=-\frac{\varepsilon c}{2 \Delta x} \frac{\ln g}{g^{N}-1} \ln R(0)
$$

where $\Delta x$ is the spatial increment of the FDTD mesh, $R(0)$ is the normal reflection coefficient, $N$ is the number of the cells in the PML thickness, and $c$ is the velocity of EM waves in the environment concerned.

\section{Floquet Periodic Boundary Condition}

Many structures of EM interest are electrically very large and, hence, pose great difficulties for computational simulation. One approach that can be used to reduce the size of the computational task is to exploit any periodicity in the structure in one or more dimensions: this concept will be exploited here, assuming that a sample of tissue is formed from a periodic grid of biological cells. In order to perform EM analysis on these types of structure with reasonable computational time, the structures are assumed to be an infinite grid and the problem can then be reduced to a unit-cell analysis via use of the Floquet boundary condition to simulate the effect of the periodic replication.

The FDTD technique was applied to the basic structure due to its simplicity and flexibility. FDTD has already been successfully extended to incorporate the Floquet theorem for the case of normal [17], [33] and oblique incidence [34], [35] for 2-D and 3-D problems. The techniques used to combine FDTD with the Floquet periodic boundary condition can be classified into two categories, i.e., direct field methods and field transformation methods [2].

\section{Lumped-Element Membrane Model}

Cells are surrounded by a thin membrane, which is the major barrier separating the cell from its environment (normally a liquid). Since the cell needs to get nutrients in and waste out, the membrane must be able to accommodate this. Therefore, the membrane has to act as a selective barrier, allowing nutrients to pass in, but keeping out many substances harmful to the cell, and acting as a dynamic barrier medium, constantly adapting to changing environmental conditions (e.g., different concentrations of ions).

The dimensions of a biological cell are around a few tens of micrometers and the thicknesses of the membranes are in the scale of a few nanometers, strongly depending on the type of the tissue. Depending on the type of the cell, voltages in the range of 20-200 $\mathrm{mV}$ can arise across the membrane. When the cell is in a resting state, the current across the membrane averages zero, but more generally, it depends on the variation of the membrane voltage [12].

Hodgkin and Huxley gave a general description of the time course of the current that flows through the membrane of the squid giant axon when the potential difference across the membrane was suddenly changed from its steady state. The results in [12] suggest that the behavior of membrane may be represented by an electric circuit [4], [12]. Current can be carried through the membrane either by charging the membrane capacitance or by movement of ions through the nonlinear conductance in parallel with the membrane capacitance. A set of equations governing the model is given in [4] and [12].

\section{IMPLEMENTATION AND VALIDATION}

\section{A. Lumped-Element Model Implementation}

To verify the correctness of the implementation of the $\mathrm{HH}$ model within the FDTD framework, the results of the analytically computed solution have been used for comparison. The 


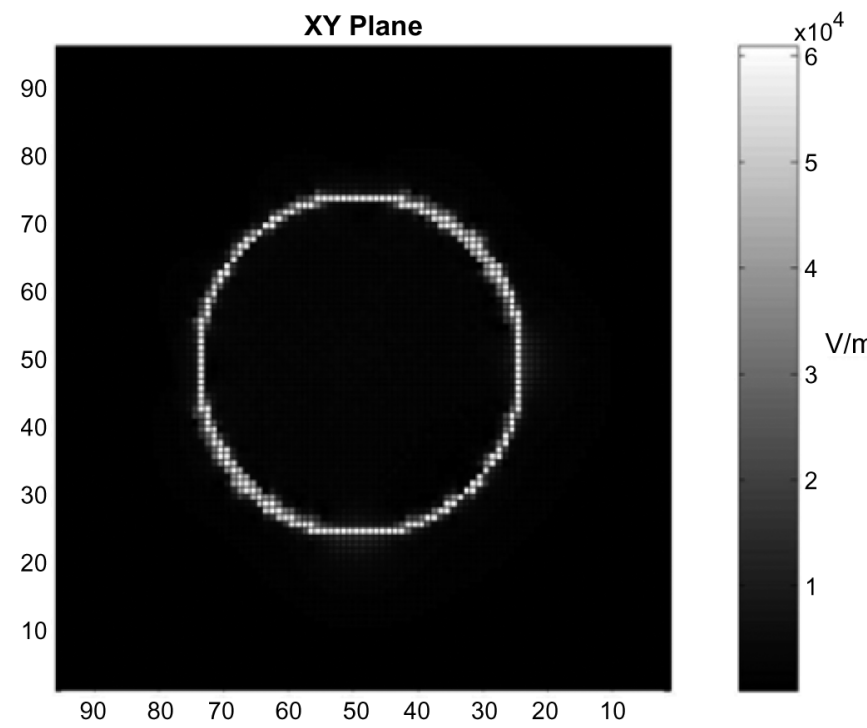

Fig. 1. Field distribution in and around a single isolated cell.

HH model was implemented on a spherical structure with diameter of $50 \mu \mathrm{m}$ and discretized with $1-\mu \mathrm{m}$ steps in order to check for the expected polarization voltage of $60.27 \mathrm{mV}$ on the membrane [4]. The HH model was included on the surface of the cell, while the regions internal and external to the sphere were considered as cytoplasm and lossy medium, respectively. It should be noted that the lumped-element FDTD method has been successfully modified in order to allow arbitrary positioning of lumped elements inside FDTD elements representing the membrane, not necessarily aligned with the FDTD grid [4], so that they represent the structure more exactly than simple FDTD. Fig. 1 depicts the expected polarization voltage of $60.27 \mathrm{mV}$ appearing on the membrane of the spherical structure without any external excitation.

\section{B. Quasi-Static FDTD Validation}

Here, a simple example will be given to illustrate this method: the results will be compared with the Mie series analytical solution [36], [37]. A two-layer sphere simulating a biological cell inside a lossy medium was considered for which the assumed properties were as follows [38]: cytoplasm (internal) $\varepsilon_{r}=48.699, \sigma=1.412 \mathrm{~S} / \mathrm{m}$; membrane $\varepsilon_{r}=11.3, \sigma=0.0 \mathrm{~S} / \mathrm{m}$; external lossy medium $\varepsilon_{r}=70.87$, $\sigma=2.781 \mathrm{~S} / \mathrm{m}$. The radius of the internal region was $25 \mu \mathrm{m}$ and the membrane thickness was set to $2 \mu \mathrm{m}$. The operating frequency was $2.45 \mathrm{GHz}$, whereas the interim transformed frequency used in this example was $30 \mathrm{GHz}$. From (1), the optimum grading factor $g$ is 6.07 for an FDTD cell size of $1 \mu \mathrm{m}$. It should be noted that this model in the FDTD computation domain is excited by a standard plane wave of amplitude $1 \mathrm{~V} / \mathrm{m}$, propagating in the $z$-direction and polarized in the $x$-direction. The field distributions along the two central axes of the layered cell are depicted in Figs. 2 and 3. As can be seen, the numerical results are in good agreement with the analytical ones. It should

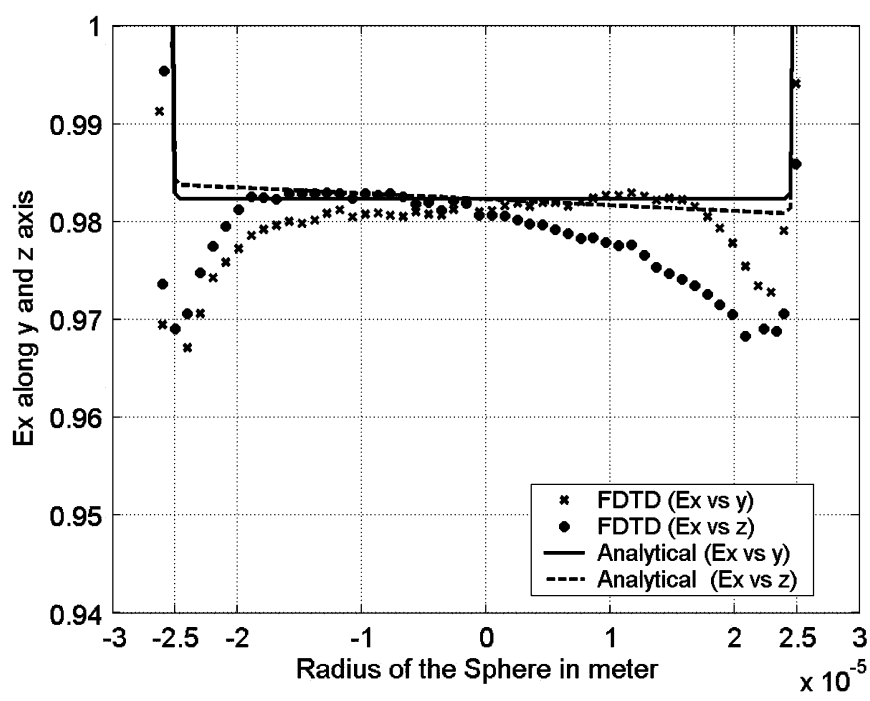

Fig. 2. Electric field $\left(E_{x}\right)$ distribution along principal axes of a double-layer sphere in lossy medium, excited by a plane wave of $1 \mathrm{~V} / \mathrm{m}$ at $2450 \mathrm{GHz}$.

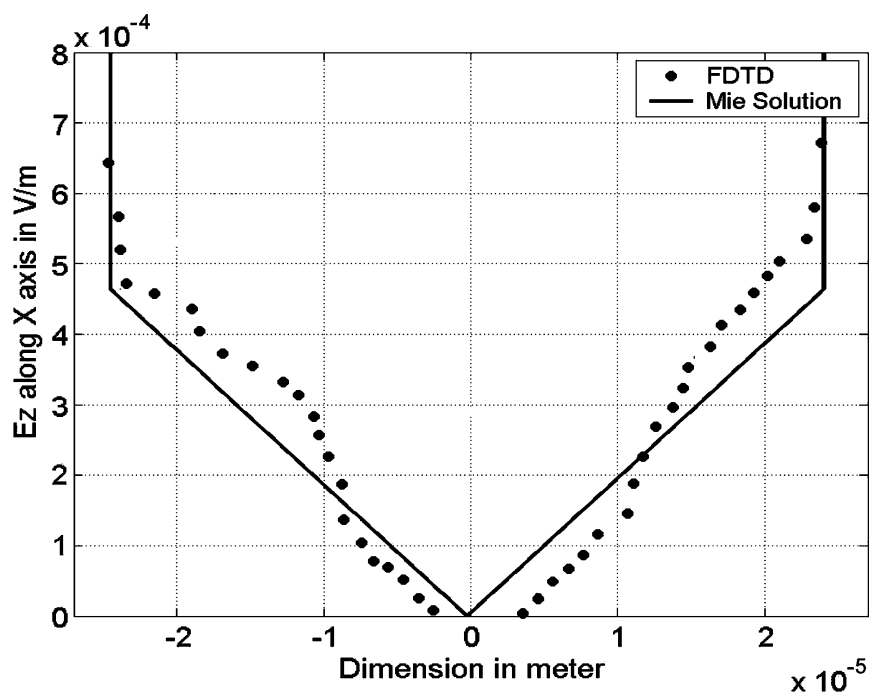

Fig. 3. Electric field $\left(E_{z}\right)$ distribution along the $x$-axis for double-layer sphere in lossy medium, excited by plane wave of $1 \mathrm{~V} / \mathrm{m}$ at $2450 \mathrm{GHz}$.

be noted that the method was executed for a total time equal to four cycles.

\section{Implementation of Floquet Boundary Condition}

The implementation of Floquet boundary conditions, quasistatic FDTD, and the current modified PML for a lossy medium excited by a $100-\mathrm{V} / \mathrm{m}$ plane wave at an operating frequency of $900 \mathrm{MHz}$ is demonstrated here. The lossy medium properties were $\varepsilon_{r}=1.0$ and $\sigma=25 \mathrm{~S} / \mathrm{m}$. The problem space and cell sizes were $21 \times 21 \times 121$ and $10 \mu \mathrm{m}$, respectively. The Floquet boundary conditions were imposed on four sides of the lossy medium. The remaining two sides were each terminated by a PML of six cells. The analyses were performed at 10, 15, and $20 \mathrm{GHz}$ and then transferred back to the desired operating frequency of $900 \mathrm{MHz}$. As can be observed in Fig. 4, the analytical and computed results are in good agreement. 


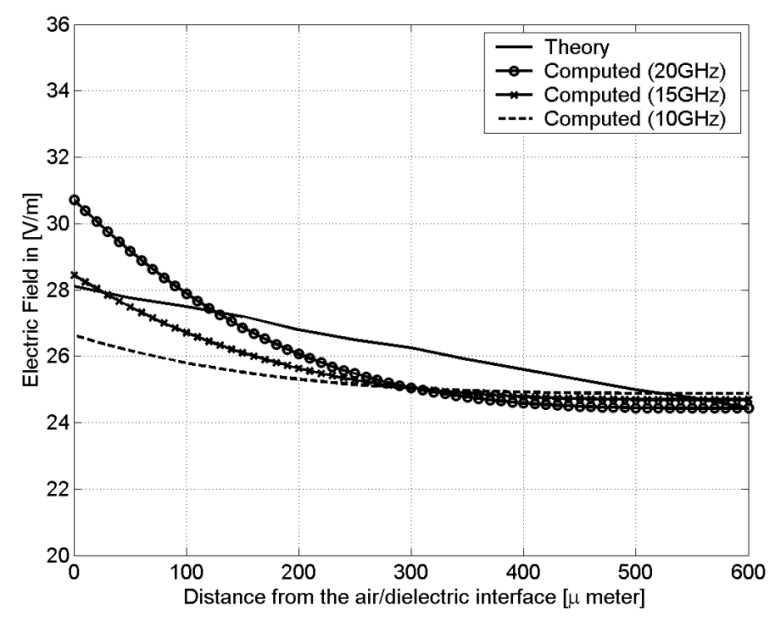

Fig. 4. Electric field along the center of the lossy medium.

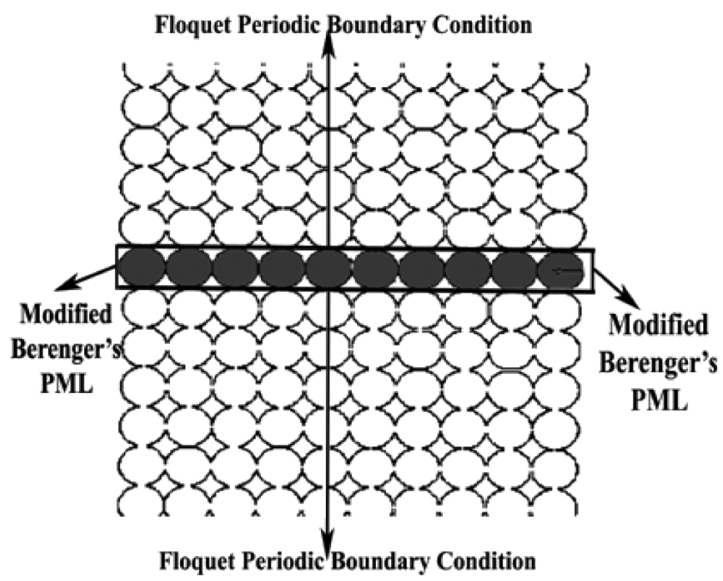

Fig. 5. 2-D view of the simulated periodic structure in the FDTD computational domain, extended by the Floquet boundary condition.

\section{Simulation AND Results}

\section{A. Connected Tissue Model Using Spherical Cells}

A stack of ten spherical cells was investigated, as shown in Figs. 5 and 6 . The radius of the each cell was $10 \mu \mathrm{m}$. The model contained three media, cytoplasm, membrane, and extracellular medium, and the dielectric properties of these were obtained from [28], as tabulated in Table I. A plane wave of $100 \mathrm{~V} / \mathrm{m}$ propagating in the $z$-direction and polarized in the $x$-direction was used as the excitation. Note that the incident plane wave excitation was applied on a plane lying between the PML region and the outer limit of the FDTD grid. In addition, in order to reduce high-frequency transients [39], [40] and dc offsets [41], [42] sometimes associated with unramped sine-wave excitations, the ramped sinusoidal source in (2) was adopted, multiplying the excitation source of $100 \mathrm{~V} / \mathrm{m}$ with the $f(t)$ functions given [41]

$$
f(t)=\left\{\begin{array}{l}
0, \quad t<0 \\
0.5\left[1-\cos \left(\omega_{r} t\right)\right] \sin \left(\omega_{r} t\right), \quad 0 \leq t \leq \frac{T_{r}}{2} \\
\sin \left(\omega_{r} t\right), \quad t>\frac{T_{r}}{2}
\end{array}\right.
$$

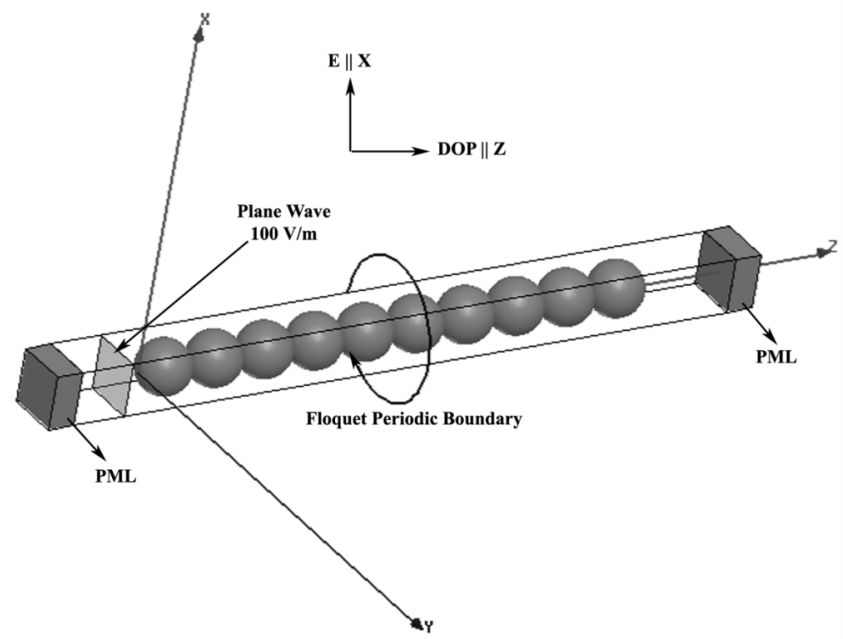

Fig. 6. 3-D view of the basic simulated spherical structures in the FDTD computational domain.

TABLE I

Electrical Properties of THE Simulated Media AT RELEVANT FREQUENCIES

\begin{tabular}{|c|c|c|c|c|}
\hline Parameter & $900 \mathrm{MHz}$ & $1800 \mathrm{MHz}$ & $2000 \mathrm{MHz}$ & $2450 \mathrm{MHz}$ \\
\hline Cytoplasm & 0.9296 & 0.9337 & 0.9344 & 0.9360 \\
Membrane & 0.9 & 0.97 & 0.9756 & 0.9838 \\
Extracellular & 0.8867 & 0.9226 & 0.9254 & 0.9301 \\
medium & & & & \\
\hline
\end{tabular}

where $T_{r}$ is the duration of the ramped cosine regime, which is approximately three source cycles.

The PML, shown in Fig. 6, was six FDTD elements wide, the grading factor $g$ was 10.1383 , and the grid structure was effectively extended to infinity in the $x$ - and $y$-directions by imposing the Floquet boundary condition along the $x$ - and $y$-axes. The Floquet periodic boundary condition plays an important role to mimic the presence of an extended 3-D structure of biological cells, simulating connected tissue. This can be easily imagined in two dimensions, as shown in Fig. 5. The FDTD problem space was $220 \times 20 \times 20$ FDTD elements of size $1 \mu \mathrm{m}$, while a discretization time step $\delta t$ of 1.3 fs was chosen to drive the FDTD computation to meet the requirements of the Courant stability criterion.

Before implementing the $\mathrm{HH}$ lumped-element model into the simulated structure, the effect of moving the Floquet boundaries gradually away from the simulated structure was studied. Figs. 7 and 8 depict the field distribution through the center of the simulated structure at $10 \mathrm{GHz}$ with varying locations of the Floquet boundaries, where $N$ cell is the number of FDTD elements between the Floquet boundaries and the boundaries of the biological cells, in the $x$ - and $y$-directions. Fig. 9 shows the field distribution on the $x z$-plane of the simulated structure for the case of $N$ cell $=10$. When the Floquet boundaries are exactly adjacent to the simulated structure $(N$ cell $=0)$, the strongest coupling effect between cells can be obtained: the highest induced field on the membrane and lowest induced field in the cytoplasm of the cell can be observed. Conversely, when the Floquet boundaries are far away from the simulated structure $(N$ cell $=10)$, 


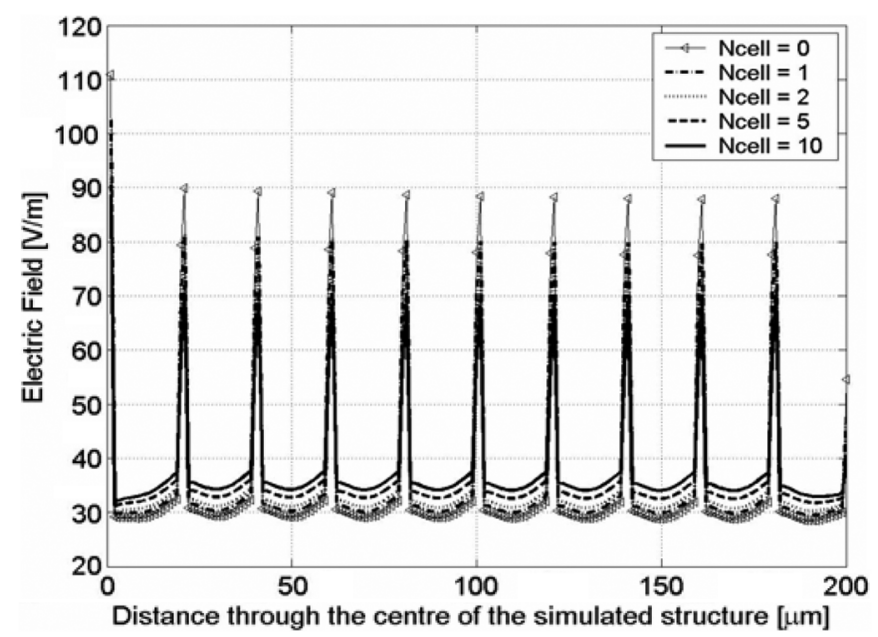

Fig. 7. Electric field distribution along $z$-axis, through the center of the simulated structure, showing effect of different spacings to the Floquet boundary condition ( $N$ cell is the number of FDTD elements from the biological cell wall to the boundary).

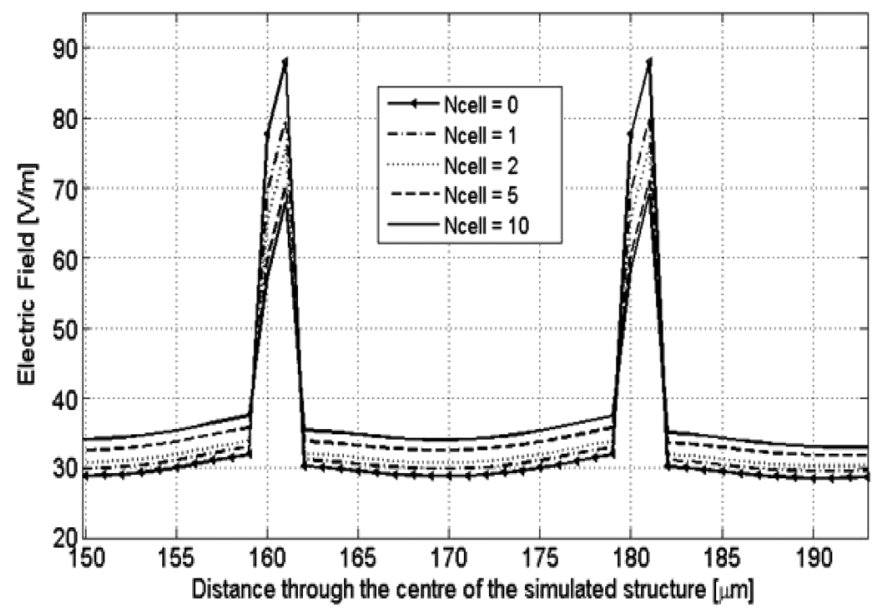

Fig. 8. Electric field distribution (enlargement of Fig. 7).

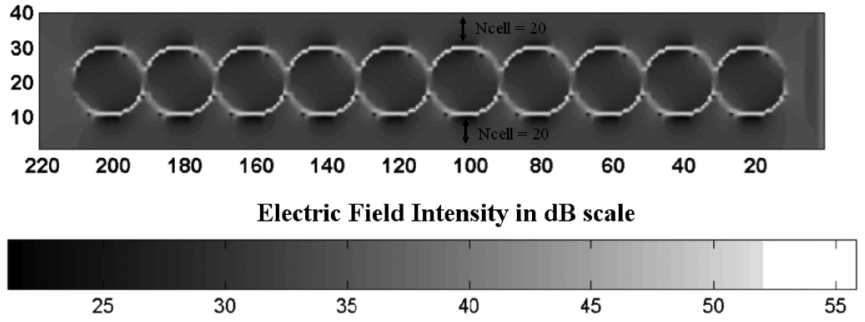

Fig. 9. Modulus of the electric field on the $x z$-plane at intermediate frequency $10 \mathrm{GHz}$, with Floquet boundary spaced 20 FDTD elements from the biological cell walls.

the lowest induced field on the membrane and highest induced field in the cytoplasm of the cell are observed. It should be noted that all the following analysis will be based on $N$ cell $=0$, which is assumed to be the most appropriate model for the real living biological tissues or cells in this microdosimetry study.

The simulations were performed at the transformed intermediate frequency of $10 \mathrm{GHz}$ and the overall model was then transformed to the intended lower frequencies. Table II reports the
TABLE II

FREQUENCY SCALING TRANSFORMATION FACTOR FROM $10 \mathrm{GHz}$ TO THE MOBILE COMMUNICATION FREQUENCIES GIVEN

\begin{tabular}{|c|c|c|c|c|}
\hline Parameter & $900 \mathrm{MHz}$ & $1800 \mathrm{MHz}$ & $2000 \mathrm{MHz}$ & $2450 \mathrm{MHz}$ \\
\hline Cytoplasm & 0.9296 & 0.9337 & 0.9344 & 0.9360 \\
Membrane & 0.9 & 0.97 & 0.9756 & 0.9838 \\
Extracellular & 0.8867 & 0.9226 & 0.9254 & 0.9301 \\
medium & & & & \\
\hline
\end{tabular}

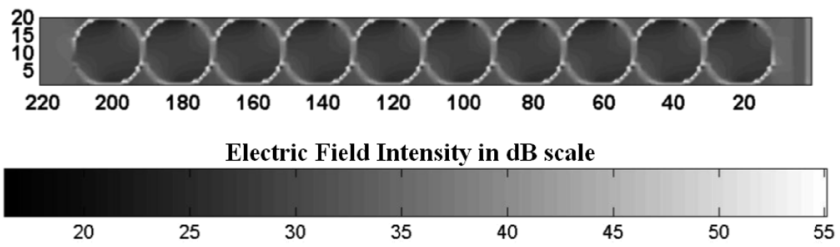

Fig. 10. Modulus of the electric field on $x z$-plane at intermediate frequency of $10 \mathrm{GHz}$ with Floquet boundary adjacent to the biological cell walls.

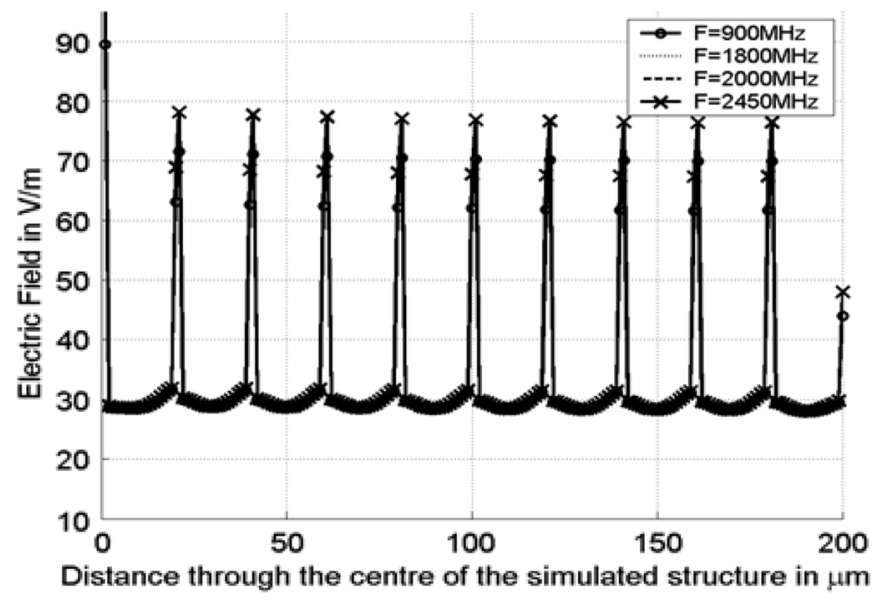

Fig. 11. Electric field distribution along $z$-axis through the center of the simulated structure in Fig. 10.

transformation factors at $900,1800,2000$, and $2450 \mathrm{MHz}$ that were used in the analysis [43].

Fig. 10 illustrates the $10-\mathrm{GHz}$ field distribution on the $x z$-plane of the simulated structure. The distributions of the electric field through the center of the simulated structure, along the incident wave propagation direction at 900, 1800, 2000, and $2450 \mathrm{MHz}$, are given in Figs. 11 and 12, where Fig. 12 is an enlarged version of Fig. 11. From inspection of Fig. 12, the field inside the cells is not constant and the induced field intensity is directly proportional to the frequency. In the other words, the higher the operating frequency used to excite the model, the higher the electric field intensity that will be induced within the analyzed structures.

To complete the simulation, the HH models were embedded in the surface of the spherical cells, in a direction normal to the surface, to represent the membrane effect of the tissue model. Versions including this were studied at frequencies of 900 and $2450 \mathrm{MHz}$. As can be seen in Figs. 13 and 14, there is a difference of approximately $15 \%$ in the field strength due to the contribution of the membrane effect from the HH model: these variations were in good agreement with expectations [4], [12], [28]. 


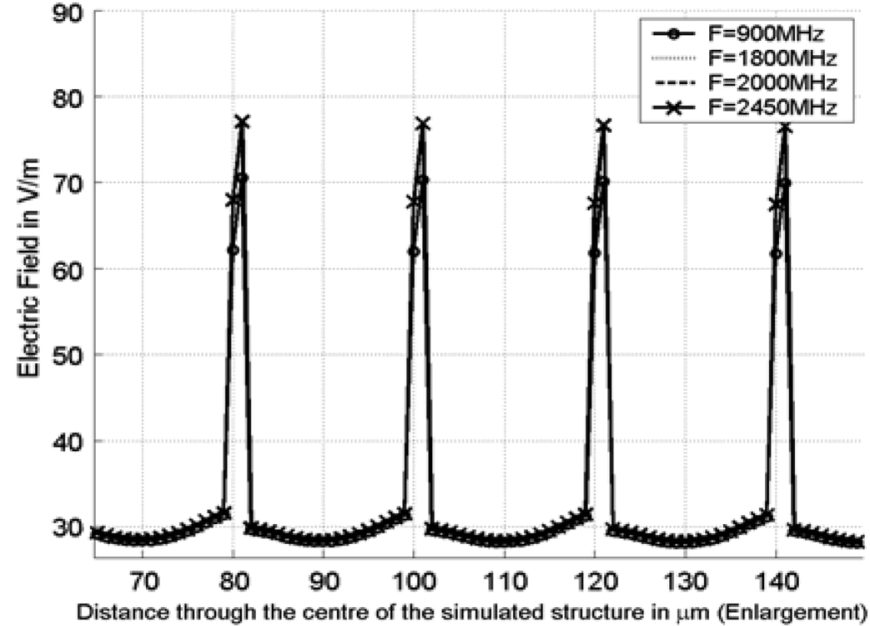

Fig. 12. Electric field distribution along $z$-axis through the center of the simulated structure in Fig. 11 (enlargement).

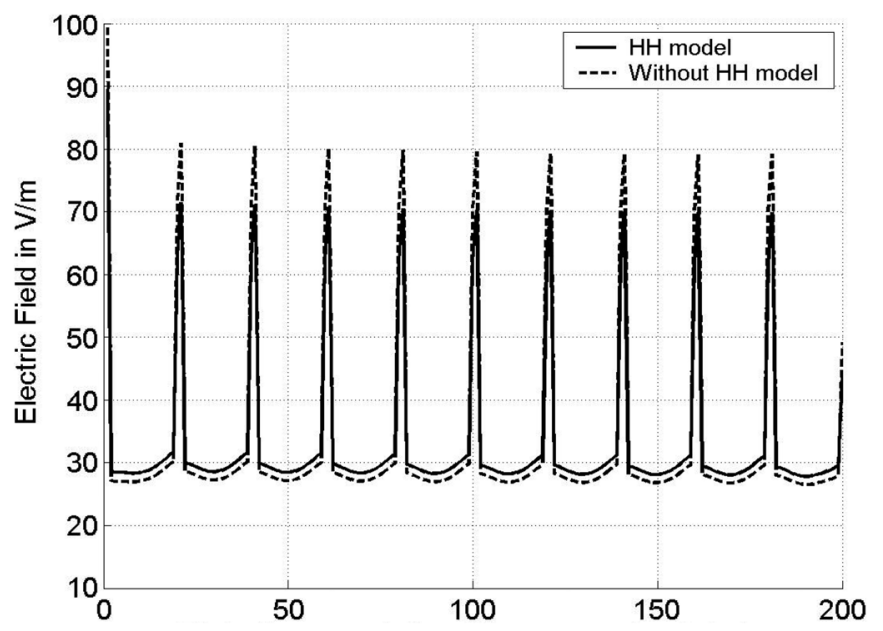

Distance through the centre of the simulated structure in $\mu \mathrm{m}$

Fig. 13. Electric field distribution along $z$-axis through the center of the simulated spherical structure in Fig. 10, incorporating $\mathrm{HH}$ model, and driven at $900 \mathrm{MHz}$.

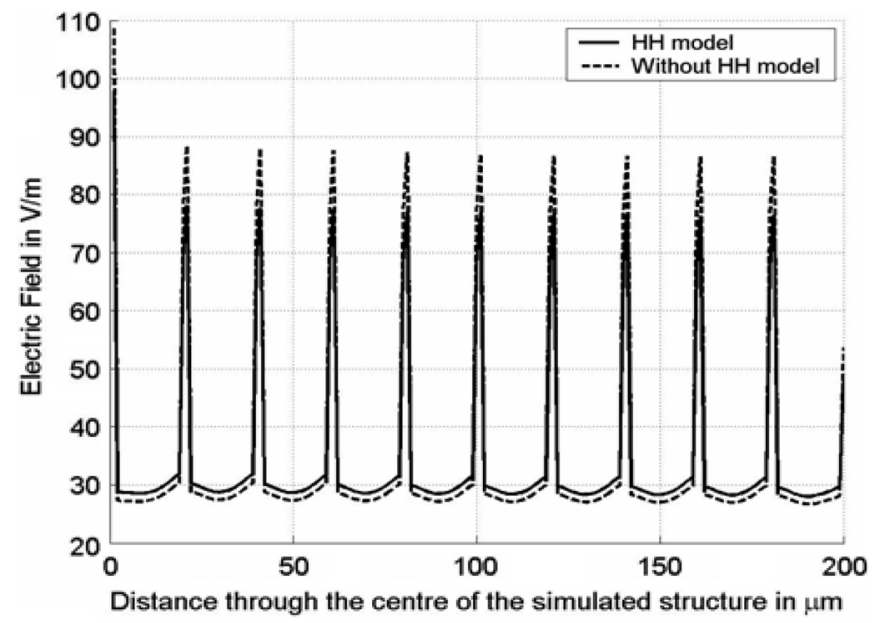

Fig. 14. Same as Fig. 13, driven at $2450 \mathrm{MHz}$.

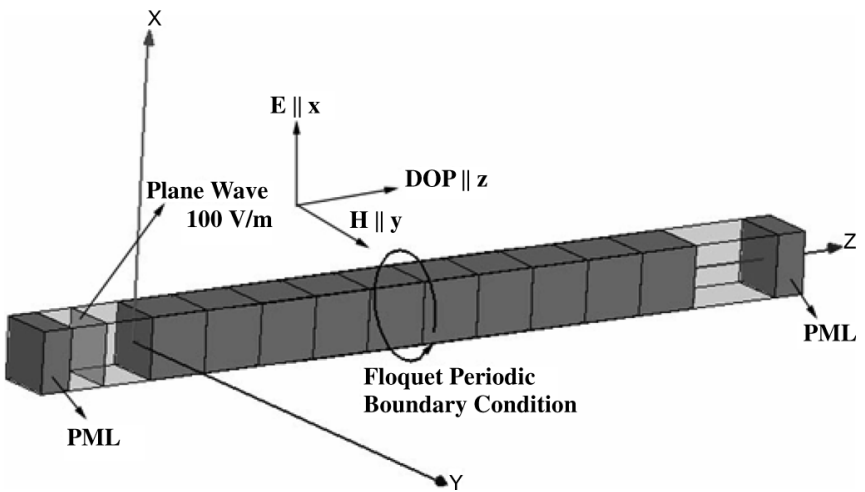

Fig. 15. 3-D view of the simulated cubical structures in the FDTD computational domain.

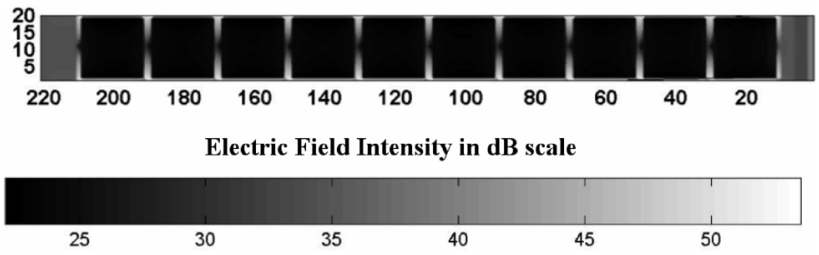

Fig. 16. Modulus of the electric field on $x z$-plane at intermediate frequency $10 \mathrm{GHz}$ with Floquet boundary adjacent to the biological cell walls.

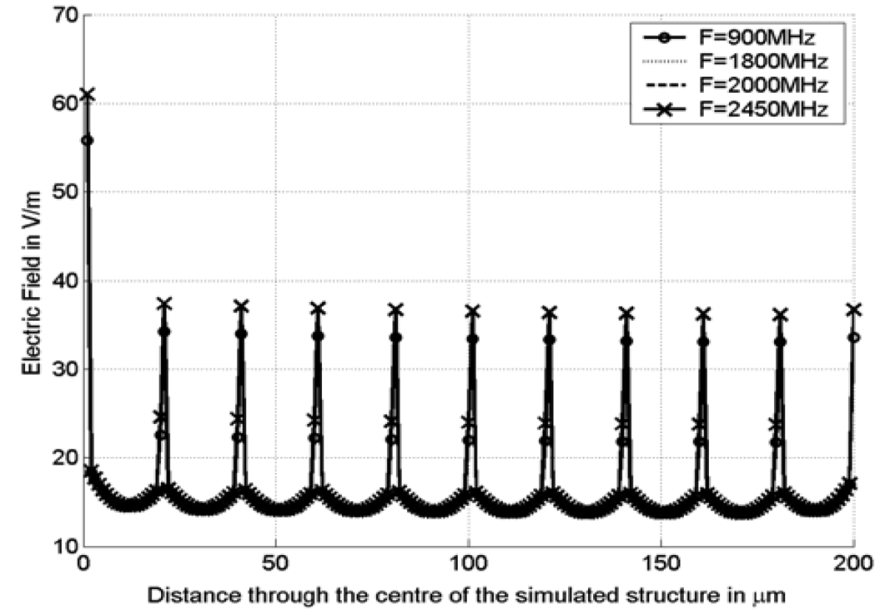

Fig. 17. Electric field distribution along $z$-axis through the center of the simulated cubical structure.

\section{B. Connected Tissue Model Using Cubical Cells}

Since living cells, when compacted into connected tissue, are not perfect spheres, a cluster of cubical cells was chosen for study on the foundation of the previous spherical-cells analysis. Fig. 15 depicts the proposed cluster of cubical cells in a 3-D view of the FDTD computational domain. In order to compare the results obtained from the previous model with this analysis, an FDTD simulation was executed, keeping the same parameter values as in the previous configuration. The 2-D view of the electric field inside the cubical-cell tissue is shown in Fig. 16. The field distributions along the propagation direction of the incident wave, through the center of the simulated structure at various frequencies, are illustrated in Figs. 17 and 18. The contribution of the $\mathrm{HH}$ model to the cubical tissue model has also been 


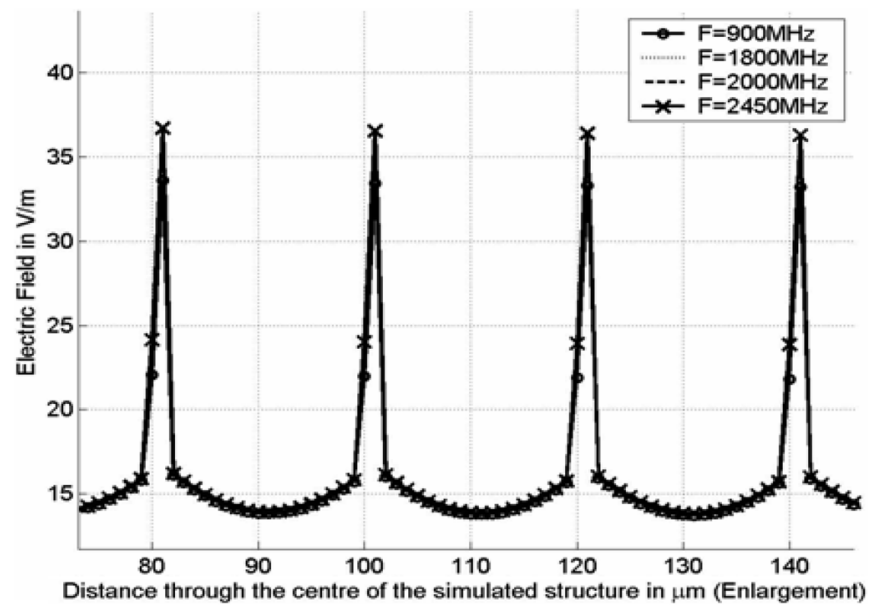

Fig. 18. Electric field distribution along $z$-axis through the center of the simulated cubical structure (enlargement of Fig. 17).

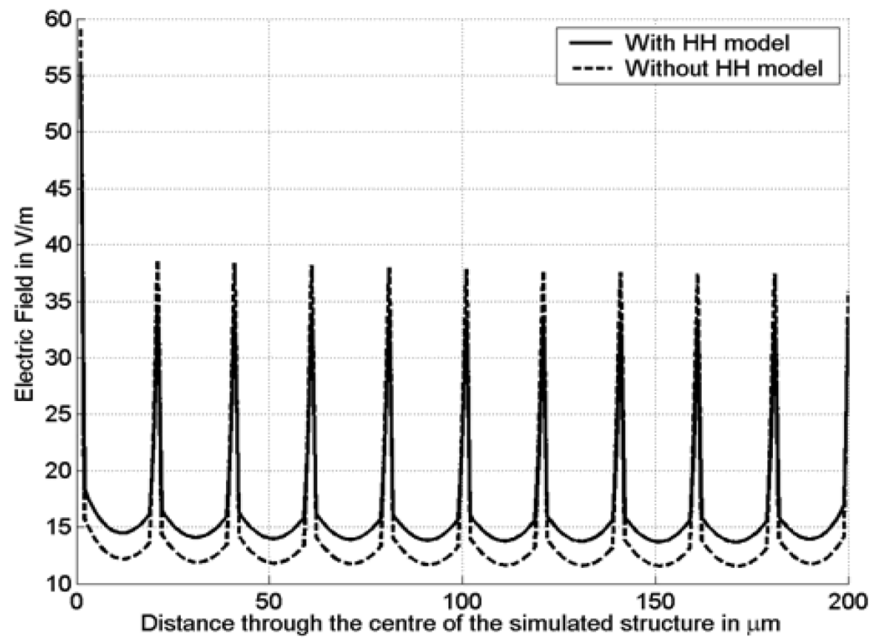

Fig. 19. Electric field distribution along $z$-axis through the center of the simulated cubical-cell structure in Fig. 16, incorporating $\mathrm{HH}$ model, and driven at $900 \mathrm{MHz}$.

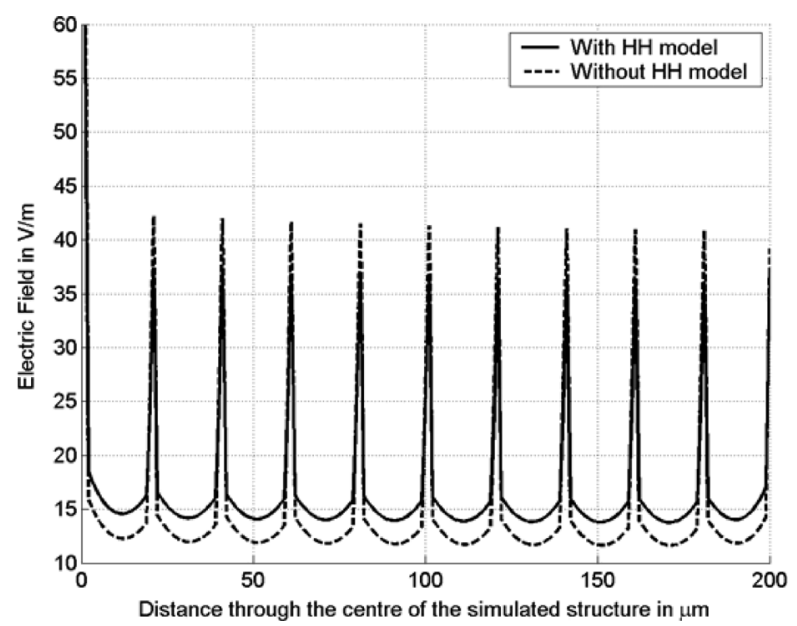

Fig. 20. Same as Fig. 19, driven at $2450 \mathrm{MHz}$.

investigated, as shown in Figs. 19 and 20. The effect of adding this model is approximately $15 \%$ difference in field, as can be seen from these figures.
The peak field on the membrane of the cubical structure is observed to be approximately three times higher than in the cytoplasm, which agrees well with the results from the structure based on spherical cells. However, the absolute field strength is approximately doubled in the spherical-cell case, presumably because of the curvature at the points studied: it is to be expected that much higher fields would be observed at the corners of the cubical cells, but it might be argued that, as a localized matter, these points do not correspond well with biological reality.

\section{CONCLUSION}

An approach to microdosimetric modeling of bioelectromagnetic interactions at the cellular level has been presented. This uses the FDTD method, combined with an arbitrarily oriented implementation of a generic lumped-element $(\mathrm{HH})$ cell-membrane model and the Floquet periodic boundary condition. By implementing a frequency-scaling approach, the number of FDTD time steps for such an electrically small structure can be reduced from several millions to a few tens of thousands. The reflection on the interface layers inside the FDTD computation domain has also been successfully reduced, even though it is within lossy penetrable media, by using a modified version of Berenger's absorbing boundary condition. The accuracy of the FDTD scaling approach was verified with idealized models of spherical cells in lossy media. The feasibility of the inclusion of the lumped-element model inside the FDTD computation domain was demonstrated. This leads to the conclusion that the application of such models allows cells of arbitrary geometries to be handled and demonstrates the viability of embedding other types of lumped-element model for membrane behavior, which is desirable since the HH model is imperfect for microwave frequencies, although it is reasonable to use it as a working hypothesis [4] to develop modeling techniques.

Use of the Floquet boundary condition enables a nontrivial region of connected biological tissue to be simulated. Such a tool will facilitate deeper investigation of the phenomena in the interaction between EM fields and biological systems at various levels of spatial definition. The combination of quasi-static FDTD with an arbitrarily oriented lumped-element membrane model, the modified Berenger absorbing boundary condition, and the Floquet periodic boundary condition represents a significant advance in verisimilitude of biological cell modeling.

\section{REFERENCES}

[1] K. S. Yee, "Numerical solution of initial boundary value problems involving Maxwell's equation in isotropic media," IEEE Trans. Antennas Propag., vol. AP-14, no. 3, pp. 302-307, May 1966.

[2] A. Taflove and S. C. Hagness, Computational Electrodynamics: The Finite-Difference Time-Domain Method, 2nd ed. Boston, MA: Artech House, 2000.

[3] O. P. Gandhi, "FDTD in bioelectromagnetics: Safety assessment and medical applications," in Advances in Computational Electrodynamics: The Finite-Difference Time-Domain Method, A. Taflove, Ed., 1st ed. : Artech House, 1998, pp. 627-632.

[4] G. Emili, A. Schiavoni, F. L. Roselli, and R. Sorrentino, "Computation of electromagnetic field inside a tissue at mobile communications frequencies," IEEE Trans. Microw. Theory Tech., vol. 51, no. 1, pp. 178-186, Jan. 2003. 
[5] O. P. Gandhi and J. Chen, "Numerical dosimetry at power-line frequencies using anatomically based models," Bioelectromagnetics, vol. 13, pp. 43-60, 1992, Suppl. 1.

[6] M. E. Potter, M. Okoniewski, and M. A. Stuchly, "Low frequency finite difference time domain (FDTD) for modelling of induced fields in humans close to line sources," J. Comput. Phys., vol. 162, pp. 82-103, 2000.

[7] L. M. Liu and S. F. Cleary, "Absorbed energy distribution from radio frequency electromagnetic radiation in a mammalian cell model: Effect of membrane-bound water," Bioelectromagnetics, vol. 16, pp. 160-171, 1995.

[8] W. Sui, D. A. Christensen, and C. H. Durney, "Extending the two-dimensional FDTD method to hybrid electromagnetic systems with active and passive lumped elements," IEEE Trans. Microw. Theory Tech., vol. 40, no. 4, pp. 724-730, Apr. 1992.

[9] M. Piket-May, A. Taflove, and J. Baron, "FDTD modeling of digital signal propagation in 3-D circuits with passive and active loads," IEEE Trans. Microw. Theory Tech., vol. 42, no. 8, pp. 1514-1523, Aug. 1994.

[10] P. Ciampolini, P. Mezzanotte, L. Roselli, D. Sereni, P. Torti, and R. Sorrentino, "Simulation of HF circuits with FDTD technique including nonideal lumped elements," in IEEE MTT-S Int. Microw. Symp. Dig., Orlando, FL, 1995, pp. 361-364.

[11] C. N. Kuo, B. Houshmand, and T. Itoh, "Full-wave analysis of packaged microwave circuits with active and nonlinear devices: An FDTD approach," IEEE Trans. Microw. Theory Tech., vol. 45, no. 5, pp. 819-826, May 1997.

[12] A. L. Hodgkin and A. F. Huxley, "A quantitative description of membrane current and its application to conduction and excitation in nerve," J. Physiol., vol. 117, pp. 500-544, 1952.

[13] A. L. Hodgkin and A. F. Huxley, "Current carried by sodium and potassium ions through the membrane of the giant axon of Loligo," $J$. Physiol., vol. 116, pp. 449-472, 1952.

[14] A. L. Hodgkin, A. F. Huxley, and B. Katz, "Measurement of current-voltage relations in the membrane of the giant axon of Loligo," $J$. Physiol., vol. 116, pp. 424-448, 1952.

[15] A. L. Hodgkin and A. F. Huxley, "The components of membrane conductance in the giant axon of Loligo," J. Physiol., vol. 116, pp. 473-496, 1952.

[16] A. L. Hodgkin and A. F. Huxley, "The dual effect of membrane potential on sodium conductance in the giant axon of Loligo," J. Physiol., vol. 116, pp. 497-506, 1952.

[17] W. J. Tsay and D. M. Pozar, "Application of the FDTD technique to periodic problems in scattering and radiation," IEEE Microw. Guided Wave Lett., vol. 3, no. 8, pp. 250-252, Aug. 1993.

[18] J. Ren, O. P. Gandhi, L. R. Walker, J. Fraschilla, and C. R. Boerman, "Floquet-based FDTD analysis of two-dimensional phased array antennas," IEEE Microw. Guided Wave Lett., vol. 4, no. 4, pp. 109-111, Apr. 1994.

[19] A. Alexanian, N. J. Kolias, R. C. Compton, and R. A. York, "Three-dimensional FDTD analysis of quasi-optical arrays using Floquet boundary conditions and Berenger's PML," IEEE Microw. Guided Wave Lett., vol. 6, no. 3, pp. 138-140, Mar. 1996.

[20] J. G. Maloney and M. P. Kesler, "Analysis of periodic structures," in Computational Electrodynamics: The Finite-Difference Time-Domain Method, A. Taflove, Ed., 2nd ed. London, U.K.: Artech House, 2000, pp. 569-625.

[21] J. Berenger, "A perfectly matched layer for absorption of electromagnetic waves," J. Comput. Phys., vol. 114, pp. 185-200, 1994.

[22] D. S. Katz, E. T. Thiele, and A. Taflove, "Validation and extension to three dimensions of the Berenger PML absorbing boundary condition for FD-TD meshes," IEEE Microw. Guided Wave Lett., vol. 4, no. 8, pp. 268-270, Aug. 1994.

[23] J. Berenger, "Perfectly matched layer for the FDTD solution of wavestructure interaction problems," IEEE Trans. Antennas Propag., vol. 44, no. 1, pp. 110-117, Jan. 1996.

[24] J. Berenger, "Improved PML for the FDTD solution of wave-structure interaction problems," IEEE Trans. Antennas Propag., vol. 45, no. 3, pp. 466-473, Mar. 1997.

[25] International FDTD databas. [Online]. Available: http://www.fdtd.org

[26] G. Mur, "Absorbing boundary conditions for the finite-difference approximation of the time-domain electromagnetic-field equations," IEEE Trans. Electromagn. Compat., vol. EMC-23, no. 4, pp. 377-382, Nov. 1981.

[27] H. Ebara, K. Tani, T. Onishi, S. Uebayashi, and O. Hashimoto, "Method for estimating complex permittivity based on measuring effective permittivity of dielectric mixtures in radio frequency band," IEICE Trans. Commun., vol. E88-B, pp. 3269-3274, 2005.
[28] T. Kotnik and D. Miklavcic, "Theoretical evaluation of the distributed power dissipation in biological cells exposed to electric fields," Bioelectromagnetics, vol. 21, pp. 385-394, 2000.

[29] W. T. Kaune and M. F. Gillis, "General properties of the interaction between animals and ELF electric fields," Bioelectromagnetics, vol. 2, pp. $1-11,1981$.

[30] A. W. Guy, S. Davidow, G. Y. Yang, and C. K. Chou, "Determination of electric current distributions in animals and humans exposed to a uniform 60-Hz high-intensity electric field," Bioelectromagnetics, vol. 3, pp. 47-71, 1982

[31] J. D. Moerloose, T. W. Dawson, and M. A. Stuchly, "Application of the finite difference time domain algorithm to quasi-static field analysis," Radio Sci., vol. 32, pp. 329-341, 1997.

[32] D. T. Prescott and N. V. Shuley, "Reflection analysis of FDTD boundary conditions-Part II: Berenger's PML absorbing layers," IEEE Trans. Microw. Theory Tech., vol. 45, no. 8, pp. 1171-1178, Aug. 1997.

[33] E. A. Navarro, B. Gimeno, and J. L. Cruz, "Modelling of periodic structures using the finite-difference time-domain method combined with the Floquet theorem," Electron. Lett., vol. 29, pp. 446-447, 1993.

[34] M. E. Veysoglu, R. T. Shin, and J. A. Kong, "A finite-difference timedomain analysis of wave scattering from periodic surfaces: Oblique incidence case," J. Electromagn. Waves Applicat., vol. 7, pp. 1595-1607, 1993.

[35] D. T. Prescott and N. V. Shuley, "Extensions to the FDTD method for the analysis of infinitely periodic arrays," IEEE Microw. Guided Wave Lett., vol. 4, no. 10, pp. 352-354, Oct. 1994.

[36] G. Mie, "Contributions to the optics of diffusing media," Ann. Phys., vol. 25 , p. 377,1908 .

[37] J. R. Mautz, "Mie series solution for a sphere," IEEE Trans. Microw. Theory Tech., vol. MTT-26, no. 5, p. 375, May 1978.

[38] C. H. See, R. A. Abd-Alhameed, M. Mutardi, P. S. Excell, and J. Vaul, "Quasi-static FDTD scheme for electrically-small regions in free space and lossless or lossy penetrable media," in 5th Int. Computat. Electromagn. Conf., Stratford-Upon-Avon, U.K., Apr. 2004, pp. 155-156.

[39] C. M. Furse, J. Y. Chen, and O. P. Gandhi, "The use of the frequencydependent finite-difference time-domain method for induced current and SAR calculations for a heterogeneous model of the human body," IEEE Trans. Electromagn. Compat., vol. 36, no. 2, pp. 128-133, May 1994.

[40] D. H. Roper and J. M. Baird, "Analysis of overmoded waveguides using the finite-difference time domain method," in IEEE MTT-S Int. Microw. Symp. Dig., 1992, pp. 401-404.

[41] C. M. Furse, S. P. Mathur, and O. P. Gandhi, "Improvements to the finite-difference time-domain method for calculating the radar cross section of a perfectly conducting target," IEEE Trans. Microw. Theory Tech., vol. 38, no. 7, pp. 919-927, Jul. 1990.

[42] C. M. Furse, D. H. Roper, D. N. Buechler, D. A. Christensen, and C. H. Durney, "The problem of treatment of DC offsets in FDTD simulations," IEEE Trans. Antennas Propag., vol. 48, no. 8, pp. 1198-1201, Aug. 2000.

[43] C. H. See, R. A. Abd-Alhameed, and P. S. Excell, "Computational modelling of electromagnetics of biological cells using lumped elements in quasi-static FDTD," in Inst. Phys. Eng. Med. Symp., Glasgow, U.K., Sep. 7-9, 2005, pp. 81-82.

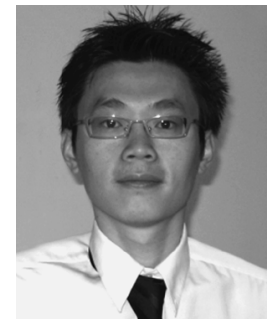

Chan H. See was born in Klang, Malaysia, in 1979. He received the B.Eng degree in electronic, telecommunication and computer engineering (with first-class honors) from the University of Bradford, Bradford, U.K., in 2002, and is currently working toward the Ph.D. degree at the University of Brandford.

From 2003 to 2006, he was with the Antennas and Applied Electromagnetic Research Group, Bradford University, where he was involved with numerous projects concentrating on antenna design and computational electromagnetics. He recently became a Research Assistant for a knowledge transfer partnership (KTP) project, to develop wireless low cost communication system for Yorkshire Water, U.K. His main research interests are in the field of computational electromagnetics, antenna design, RF circuit design and wireless sensor network.

Mr. See is a member of the Institution of Electrical Engineers (IEE), U.K. 


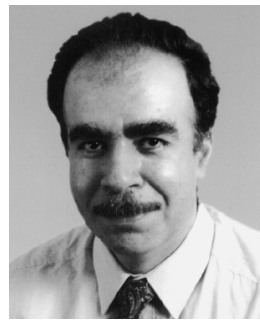

Raed A. Abd-Alhameed was born in Basrah, Iraq, in 1959. He received the B.Sc. and M.Sc. degrees from Basrah University, Basrah, Iraq, in 1982 and 1985, respectively, and the Ph.D. degree from the University of Bradford, Bradford, U.K., in 1997, all in electrical engineering.

From 1997 to 1999 , he was a Post-Doctoral Research Fellow with the University of Bradford, where he specialized in computational modeling of EM field problems, microwave nonlinear circuit simulation, signal processing of preadaption filters for adaptive antenna arrays, and simulation of active inductance. From 2000 to 2003, he was a Lecturer with the University of Bradford. In August 2003, he became a Senior Lecturer of applied electromagnetics with the University of Bradford. Since September 2005, has been a Reader of radio frequency engineering with the School of Engineering, Design and technology, Bradford University. His current research interests include hybrid EM computational techniques, genetic algorithms, multiple-input-multiple-output (MIMO) systems, antenna design, low specific absorption rate (SAR) antennas for mobile handsets, RF mixers, and active antennas.

Dr. Abd-Alhameed is a Chartered Engineer in the U.K. He is a member of the Institution of Electrical Engineers (IEE), U.K. He is a Fellow of the Higher Education Academy.

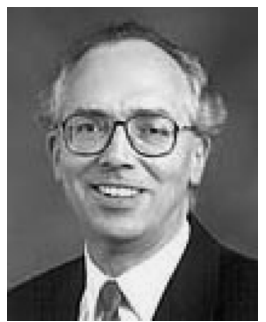

Peter S. Excell (M'80-SM'84) received the B.Sc. degree in engineering science from the University of Reading, Reading, U.K., in 1970, and the Ph.D. degree from the University of Bradford, Bradford, U.K., in 1980. His doctoral research concerned EM hazards.

He is currently a Professor of applied electromagnetics and Associate Dean for Research with the School of Informatics, University of Bradford, where he has worked since 1971. He has authored or coauthored over 200 papers. He also holds two patents. His research interests cover computational electromagnetics, electromagnetic compatibility (EMC), antenna design, bioelectromagnetics, and mobile content.

Dr. Excell is a Chartered Engineer in the U.K. He is a Fellow of the Institution of Electrical Engineers (IEE), U.K. 